\title{
Relation of synovitis, bone marrow lesions and cartilage damage to pain sensitization in people with knee osteoarthritis presenting for total knee replacement: an observational study
}

Ana Kuttapitiya, Abiola Harrison, Lena Assi, Dimitrios Christidis, Vivian Ejindu, Christine Heron, Caroline Hing, Philip Mitchell, Simon Bridle, Franklyn Howe, Nidhi Sofat

Background: Osteoarthritis $(\mathrm{OA})$ is at the most common chronic arthritic joint disease leading to pain and functional impairment. Mechanisms of pain in OA include sensitization to pain. Sensitization in $O A$ could be mediated by peripheral joint injury, leading to activation of central brain pain processing pathways. Previous studies have not shown any relation between radiographic severity of knee $O A$ and pain sensitization. The relation of $O A$ joint structural abnormalities including cartilage damage, bone marrow lesions (BMLs) and synovitis/effusions to pain sensitization, particularly in people presenting for total knee replacement (TKR), are not well understood.

Objectives: We sought to establish whether specific components of tissue injury in $O A$, namely cartilage damage, BML and synovitis, detected by magnetic resonance imaging (MRI) could provide the necessary peripheral nociceptive inputs respectively to induce pain sensitization in people presenting for TKR.

Methods: The Pain Perception in Osteoarthritis Study (PAPO) is a longitudinal cohort of people undergoing total knee replacement for knee OA (UKCRN ID 15707). Participants had WOMAC scoring, plain radiographs, MRIs (3T Phillips) of the target knee and standardised somatosensory assessment of pain pressure thresholds (PPT) before TKR and at 3, 6 and 12 months after surgery. PPT was measured with a hand held algometer (Somedic) $\left(1 \mathrm{~cm}^{2}\right.$ tip, $0.5 \mathrm{~kg} / \mathrm{sec}$ ) recorded at the point where the participant reported pressure change to pain. Recordings ( $x 3$ ) were taken at 13 points including the target knee, contralateral knee, target malleolus and radial head in the forearms. The MRI Knee Osteoarthritis Score (MOAKS) assessed the degree of synovitis, effusion, cartilage damage and BMLs. MOAKS scoring was conducted by 2 independent Consultant Radiologists and a consensus score reached. The scoring assessed presence of each lesion at a level of $1(<33 \%)$ of subregional volume (SV), 2 (33-66\%) of SV and 3 (>66\%) of SV. We evaluated the presence of synovitis/effusion, cartilage damage and BMLs and their relation to PPT when participants presented just before TKR within 6-8 weeks of surgery.

Results: A total of 60 knees were analysed with 1170 points tested for PPTs. Demographics showed a mean age 68.5 , mean BMI 32.3 and $86.7 \%$ female. The mean WOMAC pain score for the group was 306.4. All participants had a mean lesion size detected by MOAKS scoring, with a prevalence of BML size $1(66.7 \%), 2$ (30.0\%), 3 (3.3\%); synovitis/effusion grade 1 (33.3\%), 2 (36.7\%), 3 (30.0\%), cartilage damage 1 (10.0\%), 2 (56.7\%), 3 (33.3\%). There was 
no significant difference in WOMAC when subjects were grouped for severity of BMLs, synovitis or cartilage degradation. Our results (Table) show that the presence of higher severity of synovitis/effusion score was associated with a significantly higher PPT $(p<0.0001)$ i.e. less sensitized. The trend for greater severity of BMLs was associated with lower PPTs i.e. more sensitized $(p=0.72)$ but did not reach significance. Our group had globally reduced PPT's in the symptomatic/contra-lateral knee and distal sites compared with published normal control data.

Table

\begin{tabular}{|lllll|}
\hline Parameter & \multicolumn{5}{l|}{ P value } \\
\hline Gender & Female 86.7\% & Male 13.3\% & \\
\hline $\begin{array}{l}\text { Age } \\
\text { (yrs) }\end{array}$ & $\begin{array}{l}\text { Range } \\
55-87\end{array}$ & Mean 68.5 & SD +/- 9.1 & \\
\hline BMI & $\begin{array}{l}\text { Range } \\
22.9-49.1\end{array}$ & Mean 32.3 & SD +/- 6.7 & \\
\hline $\begin{array}{l}\text { Radiographic } \\
\text { scoring of BMLs } \\
\text { to Pain pressure } \\
\text { thresholds }\end{array}$ & MOAKS score & MOAKS score & MOAKS score & \\
\hline $\begin{array}{l}\text { Synovitis/ } \\
\text { Effusion }\end{array}$ & 399.0 & 2 & 3 & $<0.0001$ \\
$\begin{array}{l}\text { Bone marrow } \\
\text { lesion }\end{array}$ & 404.3 & 322.2 & 455.1 & 0.72 \\
$\begin{array}{l}\text { Cartilage } \\
\text { damage }\end{array}$ & 339.4 & 385.3 & 328.5 & \\
\hline
\end{tabular}

\section{Discussion}

People with severe knee OA presenting for TKR have globally reduced PPTs compared with non-OA populations. PPTs rose significantly with increasing inflammation, as demonstrated by high synovitis/effusion scores i.e. less sensitization, whereas PPTs had a trend to fall with increasing BML size i.e. more sensitized. Our results suggest that synovitis/effusion may represent more inflammatory components of OA pain, with BMLs contributing to sensitization components of OA pain perception. 